\title{
A PSYCHIATRIC CASE REGISTER IN NORTH-EAST SCOTLAND
}

\author{
BY
}

\author{
J. A. BALDWIN, G. INNES, W. M. MILLAR, AND G. A. SHARP \\ Department of Mental Health, University of Aberdeen
}

AND

N. DORRICOTT

Records Officer, Royal Cornhill and Associated Hospitals, Aberdeen

Much psychiatric morbidity is chronic and extends over large segments of the individual's lifespan. A major impediment to obtaining accurate and complete knowledge of the natural history of mental disorder has been the difficulty in obtaining adequate data. The case register method is potentially useful in filling this need. The essential theoretical features of the case register which distinguish it from other techniques are:

(1) Total populations can be studied as well as selected or random samples;

(2) Unlike indexes and other data-collection systems, data are individual rather than event-orientated;

(3) Data collection is systematic and standardized rather than haphazard and heterogeneous, so that the experience of each of a group of physicians serving a defined population can become the accumulated experience of all;

(4) Data are prospective and cumulative, rather than retrospective and point-related, so that studies may be designed to test specific hypotheses and adapted to changing needs and interests, and may incorporate the time perspective of longitudinal investigation.

For a psychiatrically morbid population in a geographically defined system of services and facilities, the psychiatric case register has clinical, epidemiological, and operational applications. For instance, it is known that much mental illness is longterm, but the natural history of clinical pictures in relation to the changing social context of the individual have never been described. Epidemiological studies have so far proved useful but inconclusive, largely because it has not been possible to maintain the enduring relationship with a population necessary for successive approximations to critical hypotheses. In operational terms, no clinical facility can effectively evaluate treatment procedures without knowledge of patients' prior and subsequent course and use of services. Planning of services on a regiona level is grossly restricted by lack of unduplicate counts of patient movement through and between facilities.

The concept of the psychiatric case register has been pioneered in the United States, the best known being that in operation in Monroe County, New York (Gardner, Miles, Bahn, and Romano, 1963). A more ambitious project is well advanced in Maryland (Phillips, Gorwitz, and Bahn, 1962), and there are a number of smaller schemes in existence. Other case registers are planned or operative in Australia, Canada, Scandinavia, and Yugoslavia (Bahn, 1962).

APPROPRIATENESS OF THE Register Method IN NORTH-EAST SCOTLAND

The North-Eastern Region of Scotland contains a unique and pertinent combination of features. Broadly, these may be expressed as a geographical and service entity, a particular stability of its population, and a marked integration of its psychiatric facilities.

The boundaries of the Region are defined by natural geographical features. Sea to the north and east and mountainous ranges to the south and west enclose some 4,400 square miles of marginal and mixed arable land. The uneven quality of its soil, the 
climate, the lack of natural resources, and its distance from major industrial and world distribution centres have contributed to a restriction of the population of the Region to a present 490,000 . Excepting a few small agricultural and fishing townships, the university city of Aberdeen, with approximately 185,000 inhabitants, is the only large population centre. From Aberdeen radiates the communication network of the area.

In a very real sense the regional boundaries mark out a circumscribed economic and social unit. Within this unit the population is occupationally spread between agriculture, fishing, light industry, and commerce, and fairly evenly divided between urban and rural dwelling. In the midst of marked population movement in other parts of Britain the population of this Region remains relatively stable. The 1951 Census showed that 86 per cent. of the population was born in the Region whilst a further 9 per cent. was born in other parts of Scotland.

The characteristics of the psychiatric services have been detailed elsewhere (Millar, Innes, and Sharp, 1962). There are 2,122 staffed adult psychiatric and 236 staffed adult defective beds. About 3,000 adult out-patients are seen annually, 90 per cent. of them at the Ross Clinic in Aberdeen. Facilities for children include 120 staffed defective beds and a psychiatric out-patient clinic at the Royal Aberdeen Hospital for Sick Children. Integration of all psychiatric services in the Region at an administrative level is heightened by the policy of sharing facilities and rotating junior and intermediate staff through many of the units. The nature and extent of the psychiatric facilities in the Region contribute to a very high incidence rate (Innes and Sharp, 1962). Only about 3 per cent. of patients living in the area go outside it for treatment, almost all to two centres bordering north and south.

These features of north-east Scotland are relevant to epidemiological, clinical, and operational research. Its natural boundaries and defined population provide a stable foundation for any population or service study. Its diversified demographic and occupational distribution makes possible the assessment of relationships between social group characteristics and the distribution of psychiatric morbidity. The lack of population mobility makes the tracing and long-term follow-up of patient groups relatively easy. Indeed, even total life history processes of mental illness can be studied. The integration of psychiatric services provides a potential for large-scale complete and unduplicated patient counts, their movement through and use of a comprehensive psychiatric service complex.

Partly as a result of these considerations, a number of research projects have been developed over the last few years. An epidemiological study of one year of referral from the Region to adult psychiatric services has been completed (Innes and Sharp, 1962). This identified group forms a cohort which is being followed over a 5-year period. Current research includes operational studies of national and regional psychiatric services (Baldwin, 1963), the role of particular facilities within the service system, and the problems involved in meeting the needs of specific patient groups, such as children and the aged. A study has also been started on some aspects of the community processes involved in a patient coming into psychiatric care.

To satisfy the research, clinical, and administrative requirements, the need became apparent for continuous, prospective, systematic data collection yielding a comprehensive and longitudinal picture of total treated psychiatric morbidity in the Region. The register method seemed especially appropriate to the characteristics of the area, its services, and its needs.

\section{The Case Record as a Research ToOl}

The advent of a new Records Department to serve the Aberdeen City Group of psychiatric services (the first of its kind in Scotland) encouraged the development of a unified group records system. The opportunity of designing a new case record suggested the possibility of its use as a vehicle for a comprehensive data-collection system, integrating the functions of clinical record-keeping, administration, and research. If the necessary rigour of systematic data-collection could be introduced and maintained, and an information search and retrieval facility incorporated, the clinical record would assume a value and importance far beyond the individual patient record. Not only would the clinician have a standard record containing a wider range of more reliable information than hitherto, but also the varying data needs of the population research programmes could be met, and a greater variety of administrative and operational information could be provided, such as routine statistical reports. Perhaps in the long run even more important, the obstacle to good clinical research of inadequate, unreliable, and inconsistent data would be removed.

One of us (N.D.), co-operating with clinical and research staffs, evolved the standard case record and necessary indexes and registration systems which are a normal part of the unit system for medical records. Five duplicate forms were designed as the basis for the case record itself, to contain all the systematic information collected. The duplicate copies were 
required for the data storage, retrieval, and processing system, run separately by the research group.

(1) Patient Contact Summary Form.-The documentation of the patient begins with the transcription to this form of brief identification details of the patient received from the referral source. This permits an immediate visual search for any previous record of psychiatric care. After the patient has been seen by a psychiatrist, diagnoses, disposal, and inservice transfers are noted on this form as they occur. An additional Contact Summary is opened for each new regional service contact.

(2) Original Patient Detail Form.-This is more comprehensive and is completed by one of a special staff of interviewers, with the patient, immediately before his first psychiatric consultation. This form contains considerable identifying information and service data as well as a comprehensive statement of previous and current medical and psychiatric history.

(3) Social Data Form.-This is also completed in interview with the patient and contains a lengthy inquiry into socio-cultural factors. Beyond its clinical function and standard epidemiological interest, effort has been made to explore fresh concepts in a wider socio-cultural field.

(4) Discharge/Transfer Summary Form.-This is completed when a patient leaves the service system by death, transfer, or discharge. It contains a brief synopsis of the treatment, discharge diagnoses, and disposal recommendations.

(5) Domiciliary and Emergency Contact Form.Many patients are seen for the first time as emergency cases in their homes and in the general hospital, and on these occasions a special shortened form, to embrace certain key data, is used. This necessary limitation of data is not critical since 80 per cent. of these patients enter other parts of the service immediately, and a high proportion of the remainder at a later date, when complete information can be gathered.

Operation of the register began with a census of adult patients in treatment at midnight on December 31, 1962. A 100 per cent. regional coverage of inpatients and 90 per cent. coverage of out-patients was obtained, totalling approximately 4,000 cases. Since that date, all in-patient admissions throughout the Region and all but 10 per cent. of out-patients and other types of service contact have been registered, though shortage of staff has prevented the use of the full set of standard forms in some of the hospitals. A complementary register system has been introduced into the Child Psychiatric Service and limited data on Local Authority Child Guidance Clinic referrals have been included.

\section{The Problem of Reliability}

Sources of error in data-collection systems of this type may be divided conveniently into five groups:

(1) Errors introduced by the patient or other informant, such as failure of memory in giving date of marriage or birth, or deliberate or unconscious falsification, such as omission of an illegitimate child;

(2) Errors due to failure of the recorder, such as mis-hearing what the patient says, misinterpreting the patient's meaning, assuming information not actually given (e.g., that a woman with a child is in fact married), misrecording, and mis-spelling;

(3) Errors introduced subsequent to the datacollection procedure, such as mis-coding, mispunching, analysis design error, and miscalculation;

(4) Errors inherent in the system design, such as inadequate record matching leading to duplication of counts on persons;

(5) Errors in clinical judgement, such as variation in diagnostic labelling.

Error types (3) and (4) are well-recognized and usually carefully avoided in good research design and procedure. Types (1) and (2) have long been virtually ignored except for rudimentary precautions but have recently received more intensive study (Cartwright, 1963). However, adequate measures to prevent their occurrence are still rarely encountered, particularly in large-scale reporting systems. For instance, reliance is often placed on nursing and auxiliary staff who are quite without knowledge of the reasons why data are required, or the uses to be made of them, and entirely without training in this unfamiliar mode of approaching patients. Data collection in these circumstances is often an added chore which may be resented unconsciously if not actively. Even clerical staff cannot be expected to comprehend the importance and relevance of items which they do not themselves have to utilize in their ordinary work. Medical staff are understandably unwilling to have their interviewing practice hampered by the requirements of a structured form, although they may well come to appreciate the freedom imparted by having factual information available before seeing the patient.

The only solution to these difficulties has seemed to us to be the employment of carefully selected persons who can be trained as interviewers to collect non-clinical data from patients and other informants. The Original Patient Detail and Social Data Forms are lengthy and to maintain a high degree of reliability a skilled interviewer takes between 20 and $\mathbf{4 0}$ minutes to complete them. For this purpose patients 
are asked to come $\mathbf{3 0}$ minutes before the scheduled initial psychiatric interview. Training in interviewing is detailed and lasts at least a month. A start is made by the trainee listening to a 90 -minute tape recording of specific instructions concerning each item on the data forms and an explanation of their purpose. After practice with the forms on colleagues, sittingin with skilled interviewers, and discussion, the trainee is ready to interview patients. Periodic monitoring by means of a one-way screen and tape recording is necessary to maintain standards. It is our experience that people drawn from outside the medical profession and its auxiliaries make the best interviewers, since they have not developed particular professional response-sets in handling and relating to patients which may impede the objective collection of data of this kind. The staff responsible for interviewing are fully conversant with all aspects of records work. They are also acquainted with the coding and processing of research data. In this way they are intimately aware of the purposes of their work, and of the special difficulties.

Type (5) errors pose a different kind of problem. Here observer variation becomes so important that there is little dispute about the necessity for special research (Kreitman, 1961). The need for some means of classifying patients by diagnosis, however crude, cannot be avoided, so that though the International Statistical Classification is currently being used, it is planned to experiment with different classification systems and to incorporate varying types of clinical data as new projects are formulated.

By these means it is suggested that the main problems of reliability may be solved, and, as machine processing procedures are developed, mechanical or electronic checking programmes can be devised to uncover error, particularly of the first four types.

\section{Register Data Handling}

For the purposes of research the principal types of data included in the forms are (1) social data; (2) patient movement data, and (3) clinical data; a fourth type pertaining to the operation of the register itself is that necessary for the identification of patients and the linkage of records. The remaining information in the five systematic data forms is administrative and clinical. These data are received from the Records Department on carbon copies of the five forms pre-printed with coding spaces. Selfcoding is limited to names and record-keeping numbers. From these forms the data are coded and punched into five 80-column cards of the ICT type. As a patient moves from one department to another, within the service, additional "event" cards are added detailing the characteristics of the move and changes in clinical state.

A patient's total psychiatric experience may be composed of a great many such events. The critical functions of the register process are to match all the records pertaining to any one individual and link them together in temporal order. This is accomplished in the ordinary unit system of medical records by manually sorting the various papers, visually matching on selected characteristics such as names, date of birth, etc., and then numbering all the papers for an individual with a common unit number. These procedures work quite well if the system is properly designed and operated, and provided the number of records is quite small, since the records staff accumulate a considerable personal knowledge of the case records which enables matching even with incomplete and unreliable information. However, when the case-load is too large to be memorized easily by the records staff, error inevitably increases, though its extent cannot be measured and correction is haphazard. Semi- or fully-automated matching and checking devices have been developed which overcome these drawbacks (Phillips and others, 1962). The psychiatric records system in this Region uses an improved form of visual matching and checking, and unit or register numbers are allocated by the Records Department. But the punch-card system permits more systematic checks of semiautomatic type. Several experiments with different techniques are being carried out using a sorter and tabulator as aids to clerical matching, and it is hoped that this experience will eventually facilitate introduction of fully automatic procedures using a computer.

Chronological ordering of the recorded events in an individual's history is achieved by means of an 8-digit Contact Number, adapted from the method used in the Monroe County register (Miles, Gardner, and Iker, 1961). In this code, the first two digits indicate the facility the patient is attending at any time. The third digit signifies whether he is an inpatient, out-patient, or day-patient, etc. The next two digits indicate the numerical position of the contact in relation to other facilities attended during the total psychiatric treatment experience. The final three digits are concerned with the present count of treatment referrals during the patient's life.

The punch-card system can be used to produce routine statistical reports or research analyses in the usual way. New data for special projects can be collected by including an appropriate form in the collection system which can be filled in by clinical, records, or research staff as required. The new data can be added to existing punch cards using columns 
reserved for the purpose, or by adding cards. Data already in the system can be related to added data during processing. Thus, for instance, investigation of a clinical problem is facilitated by having already available very complete demographic and social information together with present and past psychiatric experience for any group of patients. It is believed that this will stimulate better based and more systematic clinical and other research. Already several clinical projects have been initiated, including a study of mobility in aged mental patients; an extensive investigation of the patient disposal patterns of psychiatrists; a controlled study of patients experiencing "blackouts" as part of their clinical picture; and a pilot project on drug prescription, administration, and side-effects in psychiatric out-patients.

\section{CONCLUSIONS}

The possibilities opened up by the development of advanced data reporting systems in psychiatric population research are manifold and far-reaching. Some of the more immediate advantages for epidemiological and operational research have been mentioned, and the usefulness of readily available, well-documented samples for many types of clinical, biochemical, and neurophysiological research will be apparent. In addition, linkage with vital data (Newcombe, James and Oxford, 1957; Acheson, 1963), would greatly facilitate genetic research into psychiatric disorders. The growth of similar registers in culturally distinct and operationally diverse geographical areas allows scope for both complementary and comparative research into the social concomitants of deviant behaviour, and this possibility has been kept very much in mind in the NorthEast Region project.

In the wider context of its many applications, the psychiatric case register represents a methodology appropriate to the concepts of the experimental area and the population laboratory which are becoming recognized as necessary tools for further advances in psychiatric research.

\section{REFERENCES}

Acheson, E. D. (1963). "Oxford Record Linkage Study: A Central File of Morbidity and Mortality Records for a Pilot Population". Oxford Regional Hospital Board, Oxford.

Bahn, A. K. (1962). Publ. Hlth Rep. (Wash.), 77, 1071.

Baldwin, J. A. (1963). Scot. med. J., 8, 227.

Cartwight, A. (1963). Milbank mem. Fd Quart., 41, 5.

Gardner, E. A., Miles, H. C., Bahn, A. K., and Romano, J. (1963). Arch. gen. Psychiat., 9, 369.

Innes, G., and Sharp, G. A. (1962). J. ment. Sci., 108, 447. Kreitman, N. (1961). Ibid., $107,876$.

Miles, H., Gardner, E. A., and Iker, H. (1961). Paper presented to 11th Annual Conference of Mental Hospital Statisticians. New Orleans, Louisiana.

Millar, W. M., Innes, G., and Sharp, G. A. (1962). In "The Burden on the Community". Nuffield Provincial Hospitals Trust, Oxford University Press, London.

Newcombe, H. B., James, A. P., and Oxford, S. J. (1957) "Family Linkage of Vital and Health Records". Atomic Energy of Canada Ltd., Chalk River, Ontario. Phillips, W., Jr., Gorwitz, K., and Bahn, A. K. (1962). Publ. Hlth Rep. (Wash.), 77, 503. 